\title{
Situation-specific theory of surgical site infection-related stimuli in patients undergoing heart transplantation
}

\author{
Rafael Oliveira Pitta Lopes*1, Rodrigo Nogueira da Silva ${ }^{2}$, Genesis de Souza Barbosa ${ }^{1}$, Caio Guilherme Silva Bias ${ }^{3}$, \\ Luciana Maria Capurro de Queiroz Oberg ${ }^{1}$, Adriana Bispo Alvarez ${ }^{1}$, Marcos Antônio Gomes Brandão² \\ ${ }^{1}$ Campus UFRJ-Macaé Professor Aloísio Teixeira, Federal University of Rio de Janeiro. Macaé, Rio de Janeiro, Brazil \\ ${ }^{2}$ Anna Nery School of Nursing, Federal University of Rio de Janeiro, Rio de Janeiro, Brazil \\ ${ }^{3}$ National Institute of Cardiology, Ministry of Health, Rio de Janeiro, Brazil
}

Received: September 18, 2019

DOI: $10.5430 /$ jnep.v10n2p82
Accepted: November 6, 2019

Online Published: November 14, 2019

\begin{abstract}
Background and objective: Heart transplant is a life-saving treatment and currently is the definitive gold-standard in the treatment of refractory heart failure. Surgical site infection is a serious complication of heart transplantation. A nursing theory predicting consequences related to the management of risk factors for surgical site infection in patients undergoing heart transplantation can be useful to nursing practice. The objective of this study was to develop a situation-specific theory of surgical site infection-related stimuli in patients undergoing heart transplantation.

Methods: It was adopted an integrative strategy to develop the theory. Multiple sources of knowledge were accessed. The Roy Adaptation Model was adopted as the foundation for the development of this situation-specific theory in a nursing perspective. A literature review on risk factors for surgical site infection in patients undergoing heart transplantation was conducted. By configuring those sources with practice expertise in a collaborative effort, risk factors for surgical site infection in patients undergoing heart transplantation were classified as contextual or residual stimuli, focal stimulus was defined, and seven theory propositions were developed.

Results: Heart transplant surgery was considered as focal stimulus. The contextual stimuli were classified as preoperative, intraoperative, and postoperative contextual stimuli. The residual stimuli were classified as preoperative and intraoperative residual stimuli. No postoperative residual stimulus was identified. Ten theory propositions were created.

Conclusions: The emerging theory can help nurses to prevent surgical site infections in patients undergoing heart transplantation. Further developments must be made in order to consider nurse-patient interactions during the prevention of surgical site infections.
\end{abstract}

Key Words: Surgical wound infection, Heart transplantation, Cardiac surgical procedures, Risk factors, Roy adaptation model

\section{INTRODUCTION}

Heart transplant is a life-saving treatment and currently is the definitive gold-standard in the treatment of refractory heart failure, demanding superior specificities and complexity to those of general heart surgeries. ${ }^{[1,2]}$ Surgical techniques, immunosuppressive regimens, diagnostic methods and approaches in the immediate and late postoperative periods make this procedure challenging in relation to healthcare. ${ }^{[3]}$

Evidence show that complications are diversified for affecting different systems of the human body, and have a multifac-

\footnotetext{
*Correspondence: Rafael Oliveira Pitta Lopes; Email: pitta_rafael@ hotmail.com; Address: 50 Aloísio da Silva Gomes Av., Granja dos Cavaleiros, Macaé, Rio de Janeiro, Brazil.
} 
torial order. ${ }^{[4]}$ Although advances have been made to control Surgical Site Infection (SSI), it remains a substantial cause of morbidity, prolonged hospitalization, and death. ${ }^{[5]}$ The situation occurs more often when using immunosuppressive regimens. ${ }^{[6]}$

SSI consists in the infection of the incision, organ, or space after the surgical procedure, and has become more frequent in patients undergoing heart transplantation than in other cardiac surgery patients, due to the presence of additional risk factors. ${ }^{[7]}$ Heart transplantation (HT) is the top priority among the thoracic operative procedure with higher risk for SSI. $^{[7]}$

The multiplicity of risk factors related to SSI tends to require the search for evidence and its articulation with clinical expertise within a theory that aids in understanding the phenomenon. In this sense, it was developed a situation-specific theory of surgical site infection-related stimuli in patients undergoing heart transplantation. Situation-specific theories are coherent representations and descriptions of specific sets of phenomena in more subscribed situations. ${ }^{[8]}$ They are more concrete than middle-range theories and are limited in the number of concepts, in the range of explanations, in the scope of research propositions, and in the outcomes claimed. In this way, situation-specific theories can answer more specific questions and can be more accessible to researchers and clinicians.

The Roy Adaptation Model (RAM) was selected to theoretically articulate the evidence about risk factors for SSI from a nursing perspective. According to the RAM, people are considered as open systems which maintain continuous interactions with the environment, being exposed to a series of circumstances, conditions, or influences that surround them and affect their adaptation responses. ${ }^{[9]}$ These conditions, circumstances, and influences that make up the environment are regarded as focal, contextual, or residual stimuli. Focal stimulus is the internal or external stimulus most immediately confronting the human system. Contextual stimuli are all other stimuli present in the situation that contribute to the effect of the focal stimulus and a residual stimulus is an environmental factor within or outside the human system with unclear effects.

The assumptions of the developing theory are: (a) SSI is a breach in the integrity of human beings with regard to the basic need of protection, so it represents a compromised adaptation level and an ineffective adaptive response; (b) Risk factors increases the likelihood of developing a disease or injury and can be semantically aligned to the concept of stimuli, by both theoretical conjectures or statistical measures of association; (c) Risk factors can be classified as contextual or residual stimuli according to the measure of association and statistical significance.

Heart transplantation has been conducted for more than 50 years, ${ }^{[10]}$ however, nursing has not yet developed a theory on the care of patients undergoing this type of surgical procedure. SSI is a possible complication of heart transplantation significantly associated with morbidity and mortality. ${ }^{[11]}$ However, nurses engage in surveillance actions that are guided by the concept of risk factor, which may be more reductionist than the concept of stimulus in the perspective of adaptation. In spite of the importance of epidemiology, there is a potential to combine clinical evidence of risk with the conceptual dimension of stimuli.

An organized, coherent and systematic articulation of statements predicting consequences related to a nursing phenomenon such as managing risk factors for surgical site infection in patients undergoing heart transplantation can be useful to nursing practice.

Theories can be described as organized, coherent, and systematic articulations of sets of statements related to discipline's phenomena and communicated as a meaningful whole. ${ }^{[8]}$ Nursing theories, in its turn, can be defined as a conceptualization of some aspect of nursing reality that can describe phenomena, explain relationships between phenomena, predict consequences, or prescribe nursing care. ${ }^{[8]}$

Theories can be classified according to their level of abstraction, goal orientation, and scope. ${ }^{[8,12]}$ Some of them are atomistic theory, grand theory, macro theory, micro theory, middle-range theory, practice theory, and praxis theory. Another type of theory that has been developed in nursing is situation-specific theory. Situation-specific theory is a kind of theory that is narrow in scope, highly concrete, and practiceoriented. It focuses on specific nursing phenomena that reflect clinical practice and that are limited to specific populations or to particular fields of practice. ${ }^{[13]}$ Because they are highly concrete and narrow in scope, situation-specific theories are easily and readily transferable to research questions or clinical situations. ${ }^{[14]}$ Thus, the aim of this study is to develop a situation-specific theory of surgical site infectionrelated stimuli in patients undergoing heart transplantation.

\section{METHODS}

It was adopted an integrative strategy to develop the theory, ${ }^{[15]}$ as represented in Figure 1. Although situationspecific theories developed on some philosophical foundations are not supposed to be testable, this situation-specific theory has its philosophical grounding on postempiricism, so some propositions can be operationalized, measured, and/or tested. 


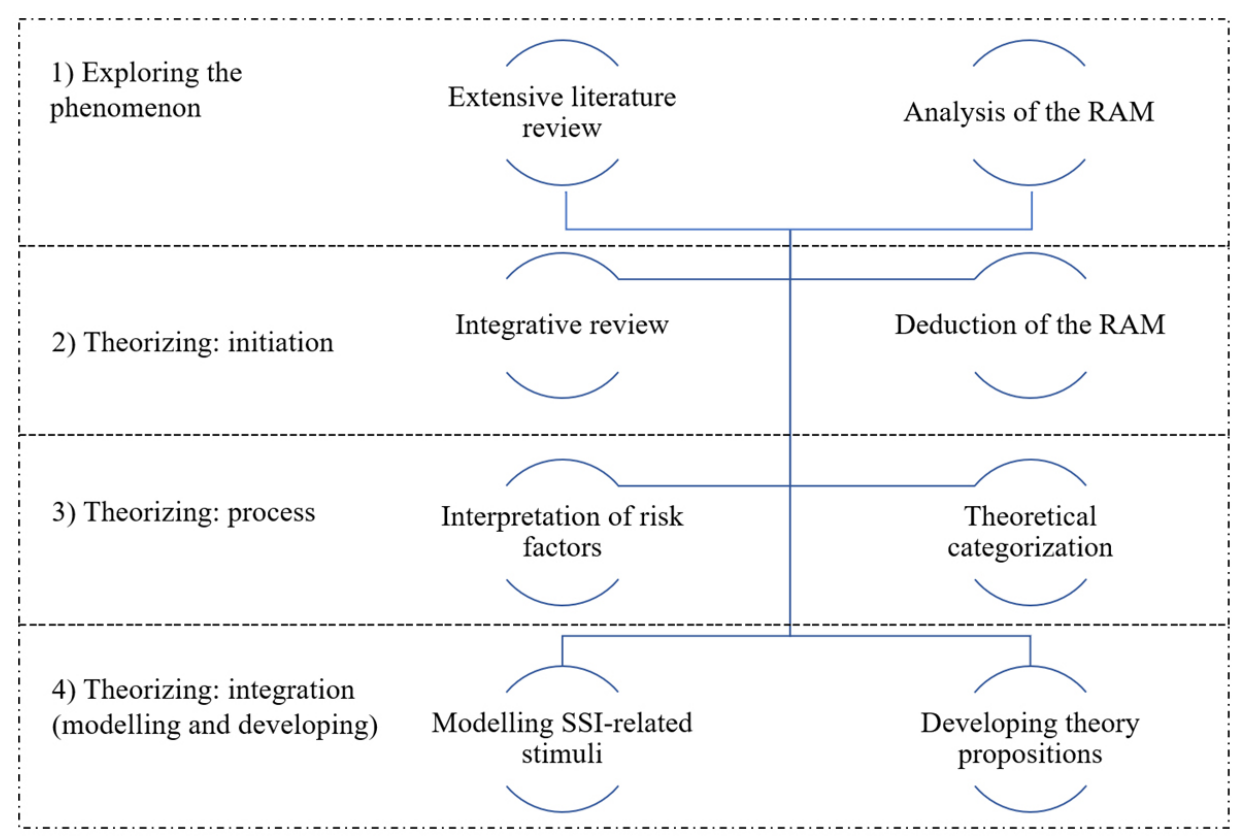

Figure 1. Integrative strategy to develop the situation-specific theory

Initially, an extensive literature review was conducted in order to explore currently existing knowledge about surgical site infection in patients undergoing heart transplantation. Then, multiple sources of knowledge were accessed by adopting the Roy Adaptation Model as the framework for the development of this situation-specific theory in a nursing perspective, by conducting an integrative review on risk factors for surgical site infection in patients undergoing heart transplantation, and by configuring those sources with practice expertise in a collaborative effort.

An integrative review was conducted in order to identify SSI risk factors. The review was carried out by following six stages: formulation of the research question, sampling of the literature, data collection, critical analysis of the included studies, discussion of the results and presentation of the integrative review. ${ }^{[16]}$

Database searches were conducted in: Latin American and Caribbean Health Sciences Literature (LILACS), Medline, Web of Science, Cumulative Index of Nursing and Allied Health Literature (CINAHL) and Scopus, in January 2018. Search terms related to heart transplantation, surgical wound infection and risk factors, both in English, in Portuguese, or in Spanish, were used to build search strategies, along with Boolean operators. 109 studies were found, 90 of them were duplicates.

The eligibility criteria consisted in: research related to the occurrence of surgical site infection in adult patients undergoing heart transplant, published between 2007 and 2017, and full-text available in English, Portuguese, or Spanish.
Book chapters, theses, dissertations, and revised or updated articles were excluded. The selection process was conducted by applying the eligibility criteria by screening the titles and the abstracts and then by screening the full-text, which was conducted by two independent reviewers with a third reviewer been consulted in cases of disagreement. Four studies were selected, as represented in Figure 2.

Critical appraisal was conducted using the Joanna Briggs Institute Critical Appraisal Checklist for Analytical CrossSectional Studies and the JBI Critical Appraisal Checklist for Randomized Controlled Trials. ${ }^{[17]}$ All selected studies have met all the critical appraisal criteria.

The following variables were collected from the studies: year, country, language of publication, directionality (prospective and/or retrospective), extension of the study (national multicenter, international multicenter or institutional), study design, data source (databases, hospital and/or prehospital records), study population, risk factors, and conclusions. Data was inserted into Microsoft Excel ${ }^{\circledR} 2010$ and submitted to a descriptive analysis and the synthesis of the results are shown in narrative form, both in Table 1 and in Figure 3.

A specific approach of deduction from theories with higher level of abstraction was applied to the Roy Adaptation Model (Theorizing: initiation stage). In the process of theorizing, the categorization of SSI-related stimuli was based on Roy's ${ }^{[9]}$ concepts of focal, contextual and residual stimuli. The heart transplantation was conceptually classified as focal stimulus (primary issue or core concept). Thus, the risk factors that interfered in the focal stimulus (heart transplan- 
tation) were classified in other stimuli types using risk measures applied in primary studies: Hazard Ratio (HR) and $\mathrm{p}$ value in univariable and multivariable Cox model; ${ }^{[18]} T$-test and Chi-squared test for difference between means and proportions of groups of Antithymocyte globulin (ATG) treated patients + Risk factor and their $p$ values; ${ }^{[19]}$ Relative Risk (RR) and $p$ value. ${ }^{[20]}$ Means or proportions higher in group with Surgical Site Infection-Related and $p<.05, \mathrm{HR}>1.0$ and $p<.05, \mathrm{RR}>1.0$ and $p<.05$ were criteria of the contextual stimuli. The risk factors classified as residual stimuli were those considered to have same criteria of the contextual stimuli, but $p$ value $>.05$. For this reason, an unclear significance of their association with SSI in HT patients was presumed. Finally, in the integration stage of theorizing, a theoretical model and theory propositions were elaborated.

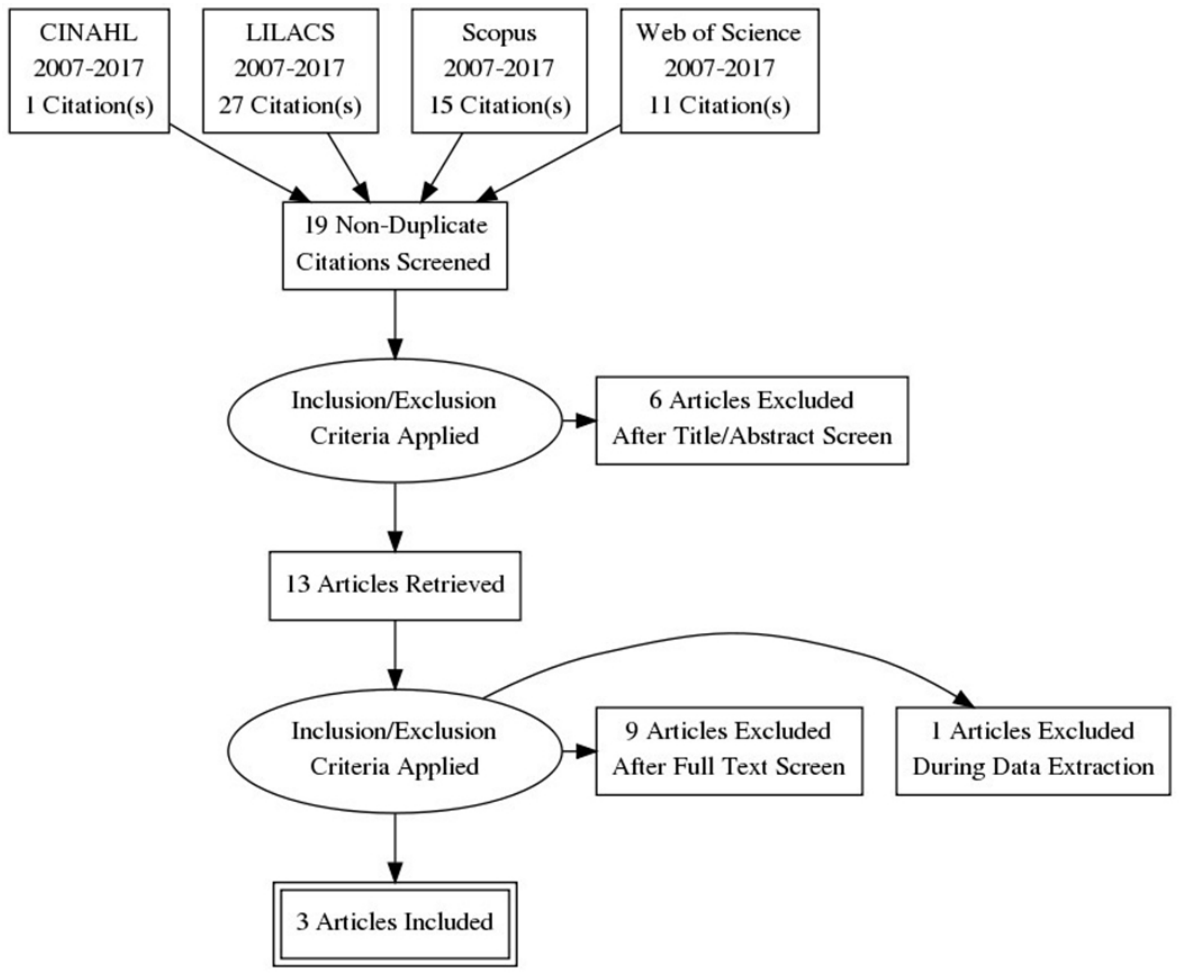

Figure 2. Preferred reporting items for systematic reviews and meta-analyses flowchart

Table 1. Main characteristics of the sample

\begin{tabular}{|c|c|c|c|}
\hline & Aim & Design & Participants \\
\hline Article $1^{[19]}$ & $\begin{array}{l}\text { To evaluate whether the ATG induction therapy in the immediate } \\
\text { post-transplant period increased the risk of infection in MCSD } \\
\text { patients with DLIs. }\end{array}$ & Cross sectional & $\begin{array}{l}57 \text { patients in the } \\
\text { postoperative period of } \\
\text { cardiac transplantation }\end{array}$ \\
\hline $\begin{array}{l}\text { Main } \\
\text { Conclusion }\end{array}$ & \multicolumn{3}{|c|}{$\begin{array}{l}\text { The use of ATG induction in patients with previous DLI does not seem to increase the risk of post-transplant } \\
\text { infection. }\end{array}$} \\
\hline Article $2^{[18]}$ & $\begin{array}{l}\text { To assess the incidence and risk factors for invasive fungal disease in } \\
\text { heart transplant recipients. }\end{array}$ & Cross sectional & $\begin{array}{l}360 \text { heart transplant } \\
\text { patients }\end{array}$ \\
\hline $\begin{array}{l}\text { Main } \\
\text { Conclusion }\end{array}$ & \multicolumn{3}{|c|}{$\begin{array}{l}\text { The incidence of fungal infectious disease was higher in the three-month period after heart transplantation, largely } \\
\text { reflecting early infection of the surgical and nosocomial sites by Candida and Aspergillus. Patients who received } \\
\text { additional immunosuppressive induction or late thoracic closure were at increased risk for fungal infectious disease. }\end{array}$} \\
\hline Article $3^{[20]}$ & $\begin{array}{l}\text { To determine the incidence, etiology, evolution and risk factors for } \\
\text { surgical site infection in the heart transplant incision. }\end{array}$ & Cross sectional & $\begin{array}{l}292 \text { cardiac transplant } \\
\text { recipients }\end{array}$ \\
\hline
\end{tabular}

Main

Conclusion

ISSIs in HT are frequently caused by resistant bacteria and Candida, but are associated with good prognosis.

Note. Abbreviations: ATG, antithymocyte globulin; DLI, driveline infections; HT, heart transplantation; ISSI, incisional surgical site infection; MCSD, mechanical circulatory support devices. 


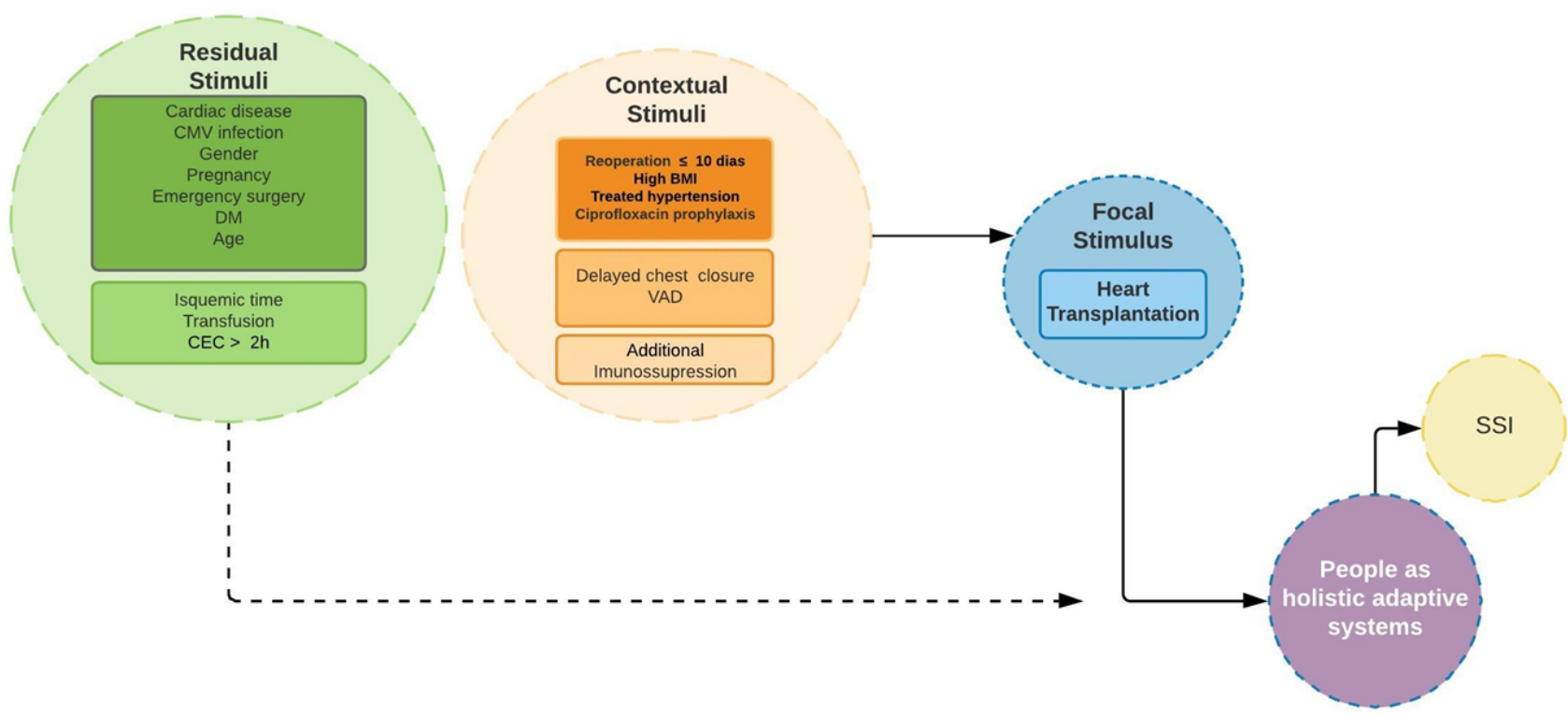

Figure 3. Theoretical model: Focal, contextual and residual stimuli of ssi in patients undergoing heart transplantation

\section{THE THEORY}

Figure 3 shows the proposed theoretical model of focal stimulus; preoperative, intraoperative and postoperative contextual stimuli; and preoperative, and intraoperative residual stimuli. This theoretical model is mainly influenced by three key concepts: Surgical Site Infection, Heart Transplant, SSI-related stimuli in HT patients.

From the evidence synthesis, it was identified that the risk factors included for investigation of surgical site infection in patients undergoing heart transplant are: heart disease; ${ }^{[18]}$ sex $;{ }^{[18-20]}$ delayed sternal closure; ${ }^{[18]}$ reoperation in less than 10 days; ${ }^{[18]}$ use of the ventricular assist device (VAD) in peri-transplantation; ${ }^{[18]}$ in Antithymocyte globulin (ATG) treated patients with high body mass index (BMI); ${ }^{[19]}$ by cytomegalovirus (CMV) infection and additional immunosuppression with OKT3, anti-thymocyte globulin or daclizumab; $;^{[18]}$ Hypertension treatment; ${ }^{[19]}$ age $;{ }^{[19]}$ blood transfusion; ${ }^{[18]}$ female gender; ${ }^{[19]}$ pregnancy; ${ }^{[19]}$ indication for transplant and ischemic time; ${ }^{[19]}$ diabetes mellitus; ${ }^{[19,20]}$ male gender; ${ }^{[19]}$ dilated cardiomyopathy; ${ }^{[20]}$ NYHA class IV; ${ }^{[20]}$ emergency type surgery; ${ }^{[20]}$ over 2 hours of cardiopulmonary bypass $(\mathrm{CPB}) ;{ }^{[20]}$ and prophylactic ciprofloxacin. ${ }^{[20]}$

Heart transplant surgery was considered as focal stimulus, because if it did not confront the human adaptation process it would not be possible for SSI to emerge as an ineffective response of this system. Furthermore, it is assumed that the surgical stimulus is the one that demands the highest amount of energy from the adaptation process of human beings, thus characterizing the focal stimulus.

The following risk factors were classified as preoperative contextual stimuli: reoperation within 10 days $(\mathrm{HR}=2.33 p$
$<.05) ;{ }^{[18]}$ ATG-treated patients with high BMI (mean $30.2 \pm$ $7.5 p<.013){ }^{[19]}$ ATG-treated patients with systemic arterial hypertension $(\mathrm{SAH})(100 \%$ cases $p<.003) ;{ }^{[19]}$ and the use of prophylactic ciprofloxacin $(\mathrm{RR}=1.1 p<.017) .{ }^{[20]}$ The intraoperative contextual stimuli were: delayed sternal closure (Univariable HR $=3.54 p=.003$; Multivariable $\mathrm{HR}=3.12 p$ $<.003$ ) ; $^{[18]}$ and use of the VAD in peri-transplantation (Univariable $\mathrm{HR}=3.54 p=0.003 p<.001) .{ }^{[18]}$ As postoperative contextual stimulus: additional induction immunosuppression (Univariable HR $=2.95 p=.01$; Multivariable $\mathrm{HR}=$ $2.68 p<.02) .{ }^{[18]}$

The risk factors classified as preoperative residual stimuli included: cytomegalovirus CMV disease $(\mathrm{HR}=1.39 p=$ $.75) ;{ }^{[18]}$ cytomegalovirus donor+/recipient $+(\mathrm{RR}=2.9 p$ $=.68) ;{ }^{[20]}$ dilated cardiomyopathy $(\mathrm{RR}=1.3 p=.76) ;{ }^{[20]}$ emergency-type surgery ( $\mathrm{RR}=1.3 p=.66) ;{ }^{[20]}$ indication for transplant and ATG-treated females with previous pregnancy $(100 \% p=.393) ;{ }^{[19]}$ male patient $(\mathrm{RR}=1.1 p=.933) ; ;^{[20]}$ ATG-treated patients with diabetes mellitus ( $66.7 \%$ cases $p$ $=.07)$; diabetes mellitus $(\mathrm{RR}=1.4 p=.58) ;{ }^{[20]}$ age mean higher $(57.3 \pm 7.9) ;{ }^{[19]}$ ATG-treated female patients (33.3\% $p=.45) .^{[19]}$ The intraoperative residual stimuli were: ischemic time, mean $(198.2 \pm 62 p=.59) ;{ }^{[19]}$ over 2 hours of $\mathrm{CPB}(\mathrm{RR}=2.6 p=.1)^{[20]}$ and transfusion $(p<.010) .{ }^{[19]}$ Postoperative residual stimuli were not identified. The NYHA class IV (Univariable HR $=0.8 p=.676)^{[20]}$ have been identified as protective factor.

\section{Theory propositions}

Considering that situation-specific theories are systematic articulations of coherent sets of propositions, ten propositions were created: 
1) Surgical Site Infection is an ineffective response of human adaptive systems who have undergone heart transplantation; 2) Heart transplantation is the stimulus (focal stimulus) most immediately confronting the human adaptation process when SSI emerges;

3) Reoperation in less than 10 days, high BMI, treatment for systemic arterial hypertension (SAH), and use of prophylactic ciprofloxacin are stimuli (contextual stimuli) that consistently can affect the focal stimulus during the preoperative period in order to provoke the human adaptive system's ineffective response (SSI);

4) Delayed sternal closure and use of ventricular assist device in peritransplantation are stimuli (contextual stimuli) that consistently can affect the focal stimulus during the intraoperative period in order to provoke the human adaptive system's ineffective response (SSI);

5) Additional immunosuppression is a stimulus (contextual stimulus) that consistently can affect the focal stimulus during the postoperative period in order to provoke the human adaptive system's ineffective response (SSI);

6) Contextual stimuli for infection may be linked to patient practices. In a person's dimension, health management and living habits (high BMI and treatment for systemic arterial hypertension) can be modified in nursing interventions;

7) Contextual stimuli may be linked to and professional dimension. Surveillance in additional immunosuppression can be modified by high care standards and by application of the protocols and guidelines;

8) Heart disease, dilated cardiomyopathy, emergency-type surgery, indication for transplant and pregnancy, diabetes mellitus, infection by CMV, age, and sex are stimuli (residual stimuli) that, during the preoperative period, have unclear significance in interfering the human adaptive system's ineffective response (SSI);

9) Ischemic time, over 2 hours of $\mathrm{CPB}$, and transfusion are stimuli (residual stimuli) that, during the intraoperative period, have unclear significance in interfering the human adaptive system's ineffective response (SSI);

10) Although in the epidemiological view, the association with infection is uncertain for residual stimuli, from the perspective of adaptation these adaptation stimuli are relevant to the clinical judgment of nurses and can be considered potentially as data of the populations at risk and associated conditions.

\section{Discussion}

From the point of view of categorization and alignment between theory and practice, it seems to have been successful to associate risk factors with the Roy Adaptation Model's concepts of stimuli. The use of the theoretical structure

Published by Sciedu Press expressed in the focal, contextual and residual stimuli, as well as representation in an iconographic model (see Figure 3), can provide a categorical description and a classification of risk factors (stimuli). These characteristics would potentially assist nursing professionals in understanding the relationships between stimuli and in adopting a more holistic evaluation and intervention perspective when dealing with the phenomenon represented by surgical site infection.

The heart transplant focal stimulus, despite not being a modifiable factor, represents a primary category of stimulus for activating the individual's adaptive system and a relevant issue to guide the clinical judgment of nursing. For the nursing diagnosis Risk for Surgical Site Infection, cardiac transplantation is incorporated into the category of associated condition as type of surgical procedure. ${ }^{[21]}$ Associated conditions are not independently modifiable by the nurse, although they can support accuracy in nursing diagnoses.

The results indicate a higher concentration of contextual stimuli in the preoperative and intraoperative periods. In the preoperative period, patients with BMI $>24.5$ had higher risk of SSI, because excess weight can alter the immune system, which in turn can lead to greater susceptibility to infections. It is necessary to point out that, despite obesity representing a contextual stimulus for SSI, morbid obesity was not configured as a risk factor for SSI, neither for mortality. ${ }^{[19]}$ The treatment of SAH, also characterized as contextual stimulus for SSI, is directly related to obesity. ${ }^{[22]}$

The occurrence of reoperation in less than ten days, identified as another contextual stimulus of the preoperative period, triggers a new focal stimulus in the ongoing response process. This situation exposes the individual to the access of new contextual stimuli, as well as reactivates previously concluded response processes.

The identification of the use of prophylactic ciprofloxacin as contextual stimulus can strengthen the contraindication of antibiotic therapy in isolation. Better results were observed when there was an association between ciprofloxacin and other antibiotics as preventive measure for SSI. ${ }^{[20]}$ However, it is necessary to consider that the analysis was based on a limited sample and its results should not be considered separately from the clinical evaluation.

Intraoperative contextual stimuli encompass procedures or situations related to surgery, being the modulators of the operative process (focal stimulus). The recognition of these situations enables the nurse to develop preliminary actions to reduce the chances of their emergence. Furthermore, understanding the procedural technologies that act as contextual stimuli for SSI allows the organization and systematization 
of nursing care focused on patient safety and on infection prevention.

Among the use of technologies, the use of VAD in peritransplantation was identified as a contextual stimulus. The use of this technology makes surgical procedures more complex, thus leading to a predisposition to fungal colonization, exposition to a fungal conidia environment and use of broadspectrum antibacterial agents. ${ }^{[18]}$ Still, regarding the intraoperative contextual stimuli, another situation detected was delayed sternal closure as stimulus for SSI caused by fungal infection. Delayed sternal closure can be understood as a situation of response to the use of technologies and consequently to the surgery's complexity. In this sub-group of Tx heart receptors, antifungal prophylaxis in peri-transplantation must be considered. ${ }^{[18]}$

Fungi can be involved in SSI after heart transplant. ${ }^{[23,24]}$ This fact may be related to prolonged hospital stay ${ }^{[25,26]}$ and to the immunosuppressive drugs prescribed to patients with transplant; ${ }^{[27,28]}$ however, evidence supporting universal antifungal prophylaxis in patients undergoing heart and lung transplantation is still inconsistent. ${ }^{[29]}$

With regard to the postoperative context, the studies obtained in the review characterized additional immunosuppression (use of corticosteroids, azathioprine and cyclosporins) as contextual stimulus. However, findings from a systematic review with meta-analysis that compared the use of interleukin-2 receptor antagonists (IL2RA) with polyclonal anti-thymocyte antibodies (ATG) during the early postoperative period showed similar risk of infections ${ }^{[30]}$ and findings from a Cochrane Systematic Review that compared immunosuppressive T-cell antibody induction with placebo, or no antibody induction, or another kind of antibody induction for heart transplant recipients showed no significant differences among any of the comparisons for infection. ${ }^{[31]}$

The evidence show that severe incisional complications were more frequent in patients using everolimus $(6.9 \%)$ compared with azathioprine $(4.2 \% ; p=.197)$ or Mycophenolate mofetil $(1.2 \% ; p=.051) .^{[19]}$ In this sense, every patient undergoing heart transplant should be monitored for signs of SSI; and professionals should be aware that the use of immunosuppression may alter some aspects of this diagnosis and of the severity of symptoms. ${ }^{[32]}$

The preoperative residual stimuli heart disease, infection by cytomegalovirus (CMV), indication for transplant, diabetes mellitus, age, female gender, male gender, pregnancy, dilated cardiomyopathy, and emergency-type surgery were supported by inconsistent results. In the intraoperative context, ischemic time, more than 2 hours of cardiopulmonary by- pass, and transfusion are residual stimuli that demand greater attention from the scientific community so more robust conclusions about the role of these factors in the occurrence of SSI may be found.

The non-identification of postoperative residual stimuli indicates the need to evaluate risk factors in the postoperative period of cardiac transplantation through exploratory studies, and then classifies them theoretically.

The theoretical propositions were elaborated by a researchtheory strategy using empirical generalizations procedures. They were constructed by hypotheses or principles and by relational propositions between two or more concepts, using evidence of clinical studies. The situation-specific theory only applies the propositions of Roy ${ }^{[9]}$ about stimuli, which reduces their connections with the propositions of the Roy Adaptation Model as a whole. However, the proposition of humans as systems in constant adaptation seems to be confirmed not by their effects, but by the risks of a need for future adaptation when facing the infection. Furthermore, the proposition of health as a reflection of the interaction or adaptation of adaptive systems seems to be valid, in the context of this situation-specific theory. ${ }^{[9]}$

\section{Limitations}

This theory lacks considerations on the sociopolitical, cultural, and/or historic contexts inherent to each nurse-patient encounter. That is due to the lack of knowledge built on this aspect of the phenomenon of interest. Further studies on the contexts involved in nurses-HT patients encounters are needed in order to refine the theory. Literature searches resulted in very few studies, which limits the strength of the claims.

\section{Conclusion}

Nurses need to be aware of the surgical site infection-related stimuli in patients undergoing heart transplantation in order to prevent death, to decrease length of hospital stay and to avoid other major complications and risks. The emerging theory can help nurses to prevent surgical site infections in patients undergoing heart transplantation by recognizing the focal, residual, and contextual stimuli that confront human adaptive systems. Further developments must be made in order to consider the contexts in which the nurse-HT patient relations are in during the prevention of surgical site infections.

The Roy Adaptation Model proved to be a theoretical structure that is able to provide conceptual categories that were compatible with the classification of scientific evidence, the risk factors of surgical site infection. The adoption of the 
theoretical concept of "stimuli" and their alignment with the clinical concept of "risk factors" seem to be an appropriate strategy to build this theory.

Heart transplant was understood as focal stimulus, being a primary category to activate an individual's adaptation system. The relevance of using a measurement of association and of the statistical significance for the categorization of risk factors as contextual and residual stimuli is emphasized. Finally, the classification of stimuli according to the operative period (preoperative, intraoperative and postoperative) can facilitate the assessment in relation to the focal stimulus, especially when applying the iconographic model proposed in the theory.

\section{FUNDING}

Rodrigo Nogueira da Silva receives financial support from the Carlos Chagas Filho Foundation for Research Support in the State of Rio de Janeiro under the Scholarship Grade 10 (Doctoral Degree), process no. E-26/200.557/2018.

\section{CONFLicts OF InTEREST Disclosure}

The authors declare that there are no conflicts of interest regarding this manuscript.

\section{REFERENCES}

[1] Mehra MR, Canter CE, Hannan MM, et al. The 2016 International Society for Heart Lung Transplantation listing criteria for heart transplantation: A 10-year update. J Hear Lung Transplant. 2016; 35(1): 1-23. PMid:26776864 https://doi .org/10.1016/j.healun.2 015.10 .023

[2] Jennings DL, Lange N, Shullo M, et al. Outcomes associated with mammalian target of rapamycin (mTOR) inhibitors in heart transplant recipients: A meta-analysis. Int J Cardiol. 2018; 265(2017): 7176. PMid:29605470 https://doi.org/10.1016/j . ijcard. 201 8.03 .111

[3] Pio F de SCG, de Azevedo DM, Marques LF, et al. Nursing care in hearth transplantation: Integrative Review. J Nurs UFPE line. 2016; 10(5): 1857-1865

[4] Coleman B, Blumenthal N, Currey J, et al. Adult cardiothoracic transplant nursing: An ISHLT consensus document on the current adult nursing practice in heart and lung transplantation. $\mathrm{J}$ Hear Lung Transplant. 2015; 34(2): 139-148. PMid:25680682 https: //doi.org/10.1016/j.healun.2014.11.017

[5] CDC Division of Healthcare Quality Promotion. National Healthcare Safety Network (NHSN) Patient Safety Component Manual. Atlanta: Centers for Disease Control and Prevention; 2019. Available from: https://www.cdc.gov/nhsn/pdfs/pscmanual/pcsman ual_current.pdf

[6] Starling P, Walsh K, Fridkin S, et al. Prevalence of HealthcareAssociated Infections in Acute Care Hospitals in Jacksonville, Florida. Infect Control Hosp Epidemiol. 2012; 33(03): 283-291. PMid:22314066 https://doi .org/10.1086/664048

[7] Berríos-Torres SI, Umscheid CA, Bratzler DW, et al. Centers for Disease Control and Prevention Guideline for the Prevention of Surgical Site Infection, 2017. JAMA Surg. 2017; 152(8): 784. PMid:28467526 https://doi.org/10.1001/jamasurg. 2017.0904

[8] Meleis AI. Theoretical Nursing: Development and Progress. 6th ed. Philadelphia: Wolters Kluwer Heath; 2018.

[9] Roy C. The Roy Adaptation Model. Upper Saddle River: Pearson Education; 2009.

[10] Hutcheson JD, Goergen CJ, Schoen FJ, et al. After 50 Years of Heart Transplants: What Does the Next 50 Years Hold for Cardiovascular Medicine? A Perspective From the International Society for Applied Cardiovascular Biology. Front Cardiovasc Med. 2019; 6(February): 1-10. PMid:30838213 https://doi.org/10.3389/fcvm. 2019. 00008

Published by Sciedu Press
[11] Wallen TJ, Habertheuer A, Gottret JP, et al. Sternal wound complications in patients undergoing orthotopic heart transplantation. J Card Surg. 2019; (November 2018).

[12] Fawcett J, Desanto-Madeya S. Contemporary Nursing Knowledge: Analysis and Evaluation of Nursing Models and Theories. 3rd ed. Philadelphia, PA: F. A. Davis Company; 2013.

[13] Im EO, Meleis AI. Situation-Specific Theories: Philosophical Roots, Properties, and Approach. Adv Nurs Sci. 1999; 22(2): 11-24. PMid:10634184 https://doi.org/10.1097/00012272-19991 2000-00003

[14] Meleis AI, Topaz M. Nursing Theory of the Future: SituationSpecific Theories. Pflege. 2011; 24(6): 345-347.

[15] Im E-O. Development of Situation-specific Theories: An Integrative Approach. Adv Nurs Sci. 2005; 28(2): 137-151. PMid:15920360 https://doi.org/10.1097/00012272-200504000-00006

[16] de Souza MT, da Silva MD, de Carvalho R. Integrative review: what is it? How to do it? Einstein (São Paulo). 2010; 8(1): 102106. PMid:26761761 https://doi.org/10.1590/s1679-45082 010rw1134

[17] Aromataris E, Zachary M. Joanna Briggs Institute Reviewer's Manual. Adelaide; 2017. Available from: https://reviewersmanual. joannabriggs.org/

[18] Rabin AS, Givertz MM, Couper GS, et al. Risk factors for invasive fungal disease in heart transplant recipients. J Hear Lung Transplant. 2015; 34(2): 227-232. PMid:25455750 https://doi.org/10.1 016/j . healun. 2014.09.036

[19] Arman D, Kuraitis D, Moriguchi J, et al. Do Prior Driveline Infections Increase the Risk of Infection in Heart Transplant Patients Treated With Rabbit Antithymocyte Globulin Induction Therapy? Transplant Proc. 2016; 48(10): 3393-3396. PMid:27931587 https://doi.org/10.1016/j.transproceed.2016.09.037

[20] Ramos A, Asensio A, Muñez E, et al. Incisional surgical infection in heart transplantation. Transpl Infect Dis. 2008; 10(4): 298-302. PMid:18482203 https://doi.org/10.1111/j.1399-3062.20 $08.00316 . \mathrm{x}$

[21] Herdman TH, Kamitsuru S. Nursing Diagnoses: Definitions and Classification. (Herdman TH, Kamitsuru S, eds.). New York: Thieme Publishers New York; 2018.

[22] Landsberg L, Aronne LJ, Beilin LJ, et al. Obesity-Related Hypertension: Pathogenesis, Cardiovascular Risk, and Treatment. J Clin Hypertens. 2013; 15(1): 14-33. PMid:23282121 https://doi.or g/10.1111/jch.12049 
[23] El-Sayed Ahmed MM, Almanfi A, Aftab M, et al. Aspergillus Mediastinitis after Orthotopic Heart Transplantation: A Case Report. Texas Hear Inst J. 2015; 42(5): 468-470. PMid:26504444 https://doi.org/10.14503/THIJ-14-4732

[24] Vallabhaneni S, Purfield AE, Benedict K, et al. Cardiothoracic surgical site phaeohyphomycosis caused by Bipolaris mould, multiple US states, 2008-2013: a clinical description. Med Mycol. 2016; 54(3): 318-321. PMid:26705838 https ://doi .org/10.1093/mm y/myv 101

[25] Prieto D, Correia P, Batista M, et al. A decade of cardiac transplantation in Coimbra: The value of experience. Rev Port Cardiol (English Ed). 2014; 33(11): 671-681. https://doi.org/10.1016/j.re pce. 2014.10 .001

[26] Ayesta A, Sousa-Casasnovas I, Gómez E, et al. Outpatient follow-up during the first year after heart transplantation, is it feasible? Int $\mathrm{J}$ Cardiol. 2017; 228: 1-3. PMid:27863348 https ://doi.org/10.1 016/j.ijcard.2016.11.188

[27] Saber-Moghaddam N, Nomani H, Sahebkar A, et al. The change of immunosuppressive regimen from calcineurin inhibitors to mammalian target of rapamycin (mTOR) inhibitors and its effect on malignancy following heart transplantation. Int Immunopharma- col. 2019; 69(November 2018): 150-158. PMid:30711744 https : //doi.org/10.1016/j.intimp.2019.01.035

[28] Kim IC, Youn JC, Kobashigawa JA. The Past, Present and Future of Heart Transplantation. Korean Circ J. 2018; 48(7): 565. PMid:29968430 https ://doi.org/10.4070/kcj.2018.0189

[29] Pilarczyk K, Haake N, Heckmann J, et al. Is universal antifungal prophylaxis mandatory in adults after lung transplantation? A review and meta-analysis of observational studies. Clin Transplant. 2016; 30(12): 1522-1531. PMid:27653443 https://doi.org/10.111 $1 / \operatorname{ctr} .12854$

[30] Briasoulis A, Inampudi C, Pala M, et al. Induction immunosuppressive therapy in cardiac transplantation: a systematic review and meta-analysis. Heart Fail Rev. 2018; 23(5): 641-649. PMid:29532201 https ://doi.org/10.1007/s10741-018-9691-2

[31] Penninga L, Møller CH, Gustafsson F, et al. Immunosuppressive T-cell antibody induction for heart transplant recipients. Cochrane Database Syst Rev. 2013; 2013(12). https : //doi.org/10.1002/ 14651858. CD008842.pub2

[32] Zuckermann A, Barten MJ. Surgical wound complications after heart transplantation. Transpl Int. 2011; 24(7): 627-636. PMid:21418335 https ://doi.org/10.1111/j.1432-2277.2011.01247.x 